\title{
Temporal evolution of small-scale internetwork magnetic fields in the solar photosphere (Corrigendum)
}

R. J. Campbell ${ }^{1}$, M. Mathioudakis ${ }^{1}$, M. Collados ${ }^{2,3}$, P. H. Keys ${ }^{1}$, A. Asensio Ramos ${ }^{2,3}$, C. J. Nelson ${ }^{1}$, D. Kuridze ${ }^{4,5}$, and A. Reid ${ }^{1}$

\footnotetext{
1 Astrophysics Research Centre, Queen's University of Belfast, Northern Ireland BT7 1NN, UK e-mail: rcampbel155@qub.ac.uk

2 Instituto de Astrofísica de Canarias, Vía Láctea s/n, 38205 La Laguna, Tenerife, Spain

3 Dept. Astrofísica, Universidad de La Laguna, 38205 La Laguna, Tenerife, Spain

${ }^{4}$ Department of Physics, Aberystwyth University, Ceredigion, Cymru SY23 3BZ, UK

5 Abastumani Astrophysical Observatory, Mount Kanobili, 0301 Abastumani, Georgia
}

A\&A 647, A182 (2021), https://doi.org/10.1051/0004-6361/202040028

Key words. methods: observational - Sun: photosphere - Sun: infrared - Sun: magnetic fields - Sun: granulation - errata, addenda

We correct a typographical error in the original paper.

Figures $10,12,14,15$, and 16 contain a table in the top right panel with four columns and four rows of values. The line of sight velocity, $v_{\mathrm{LOS}}$, values for scheme 1 inversions (in the fourth row, first column and second column) are inserted the wrong way around. The values themselves are correct, but printed in the wrong column. All other values in the tables remain unaffected, and none of the paper's discussions or conclusions are impacted. We provide corrected versions of Figs. 10, 12, 14, 15, and 16 in Figs. 1, 2, 3, 4, and 5, respectively. 
A\&A 652, C2 (2021)
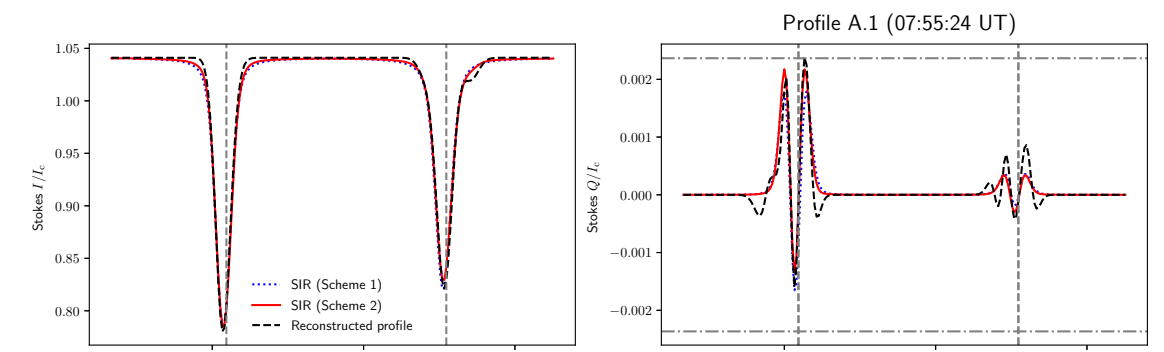

\begin{tabular}{|c|c|c|c|c|}
\hline & $\begin{array}{l}\begin{array}{l}\text { Sche } \\
(\text { model } 1)\end{array} \\
\end{array}$ & $\begin{array}{l}\text { Scheme 1 } \\
(\text { model 2) }\end{array}$ & $\begin{array}{l}\text { Schom } \\
\text { (model 1) }\end{array}$ & $\begin{array}{l}\text { Scheme } \\
(\text { model 2) }\end{array}$ \\
\hline$B[G]$ & 0 & 305 & 155 & 295 \\
\hline$\gamma$ [deg] & 0 & 82 & 86 & 18 \\
\hline$\phi[\operatorname{deg}]$ & 0 & 211 & 32 & 85 \\
\hline$v_{\text {LOS }}[\mathrm{km} / \mathrm{s}]$ & -1.2 & -1.2 & -1.4 & 3.9 \\
\hline$\alpha$ & 0.803 & 0.197 & 0.985 & 0.015 \\
\hline
\end{tabular}
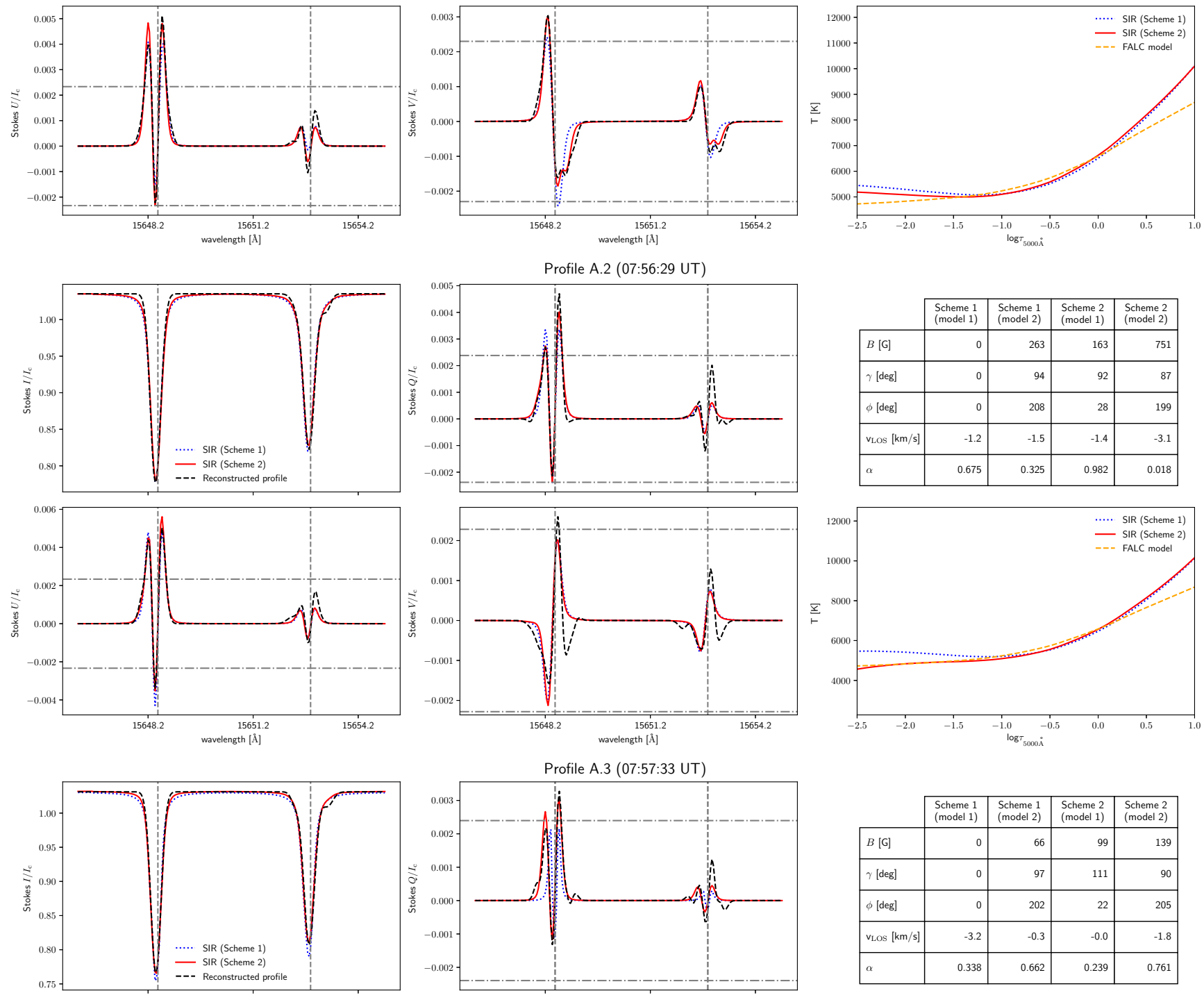

\begin{tabular}{|l|r|r|r|r|}
\cline { 2 - 5 } \multicolumn{1}{c|}{} & $\begin{array}{c}\text { Scheme 1 } \\
\text { (model 1) }\end{array}$ & $\begin{array}{r}\text { Scheme 1 } \\
\text { (model 2) }\end{array}$ & $\begin{array}{r}\text { Scheme 2 } \\
\text { (model 1) }\end{array}$ & $\begin{array}{r}\text { Scheme 2 } \\
\text { (model 2) }\end{array}$ \\
\hline$B[G]$ & 0 & 66 & 99 & 139 \\
\hline$\gamma[\operatorname{deg}]$ & 0 & 97 & 111 & 90 \\
\hline$\phi[\operatorname{deg}]$ & 0 & 202 & 22 & 205 \\
\hline$v_{\text {L.oS }[\mathrm{km} / \mathrm{s}]}$ & -3.2 & -0.3 & -0.0 & -1.8 \\
\hline$\alpha$ & 0.338 & 0.662 & 0.239 & 0.761 \\
\hline
\end{tabular}
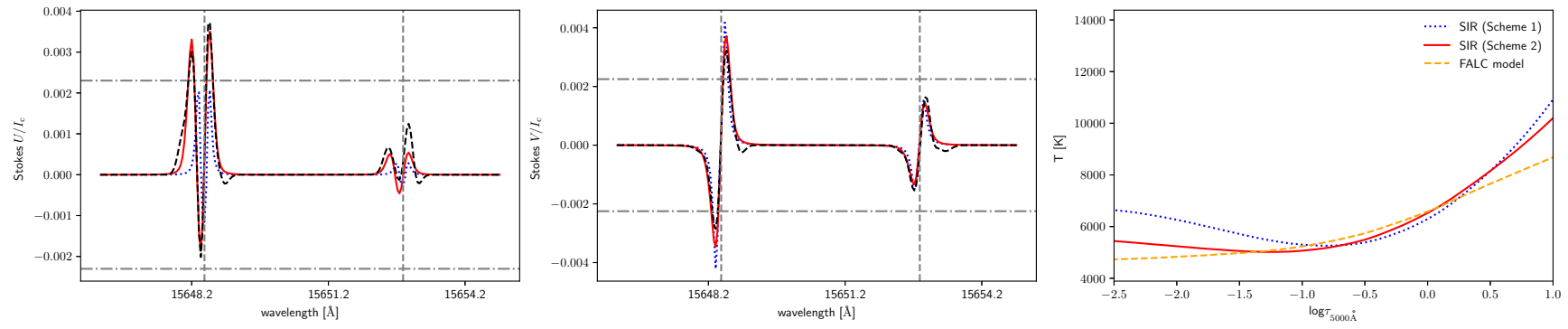

Fig. 1. Corrected Fig. 10. 
R. J. Campbell et al.: Temporal evolution of small-scale internetwork magnetic fields in the solar photosphere (Corrigendum)
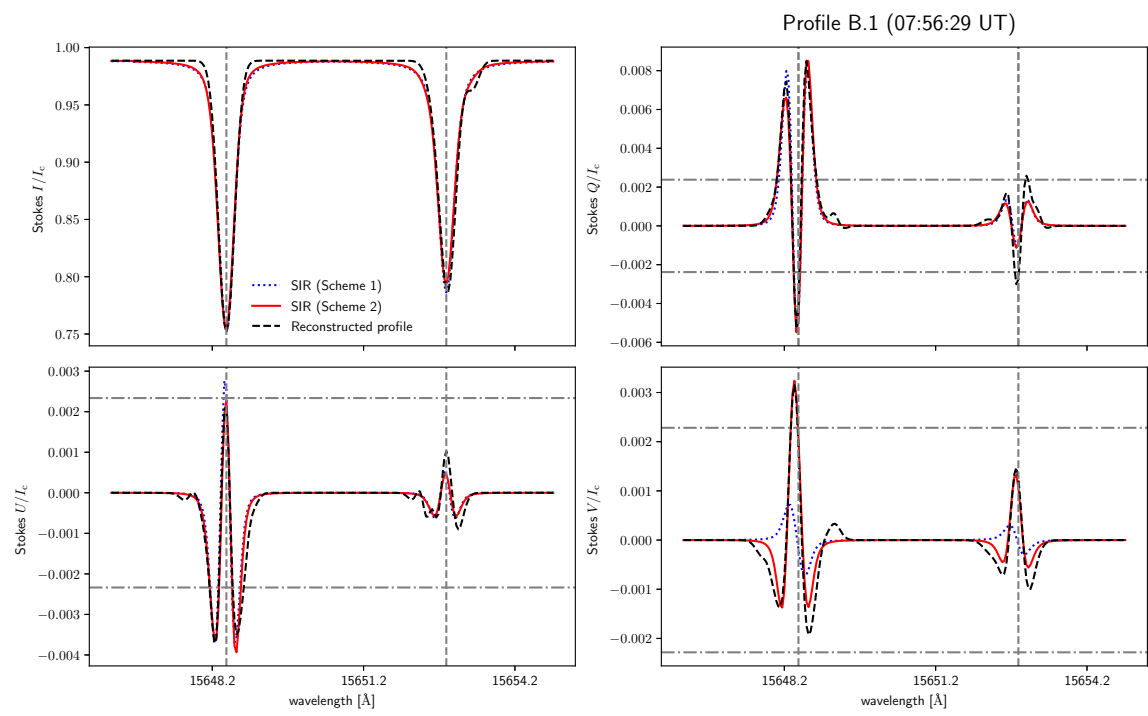

\begin{tabular}{|c|c|c|c|c|}
\hline & $\begin{array}{l}\text { Scheme 1 } \\
\text { (moded 1) }\end{array}$ & 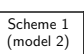 & $\begin{array}{l}\text { Scheme } 2 \\
\text { (model 1) }\end{array}$ & $\begin{array}{l}\text { Scheme e } \\
\text { (model 2) }\end{array}$ \\
\hline$B[G]$ & 0 & 283 & 209 & 422 \\
\hline$\gamma[\operatorname{deg}]$ & 0 & 88 & 84 & 103 \\
\hline$\phi[\mathrm{deg}]$ & 0 & 167 & 165 & 172 \\
\hline$v_{\operatorname{LLS}}[\mathrm{km} / \mathrm{s}]$ & 0.4 & -0.5 & 0.1 & -1.7 \\
\hline$\alpha$ & 0.529 & 0.471 & $\begin{array}{ll}0.847 \\
\end{array}$ & 0.153 \\
\hline
\end{tabular}
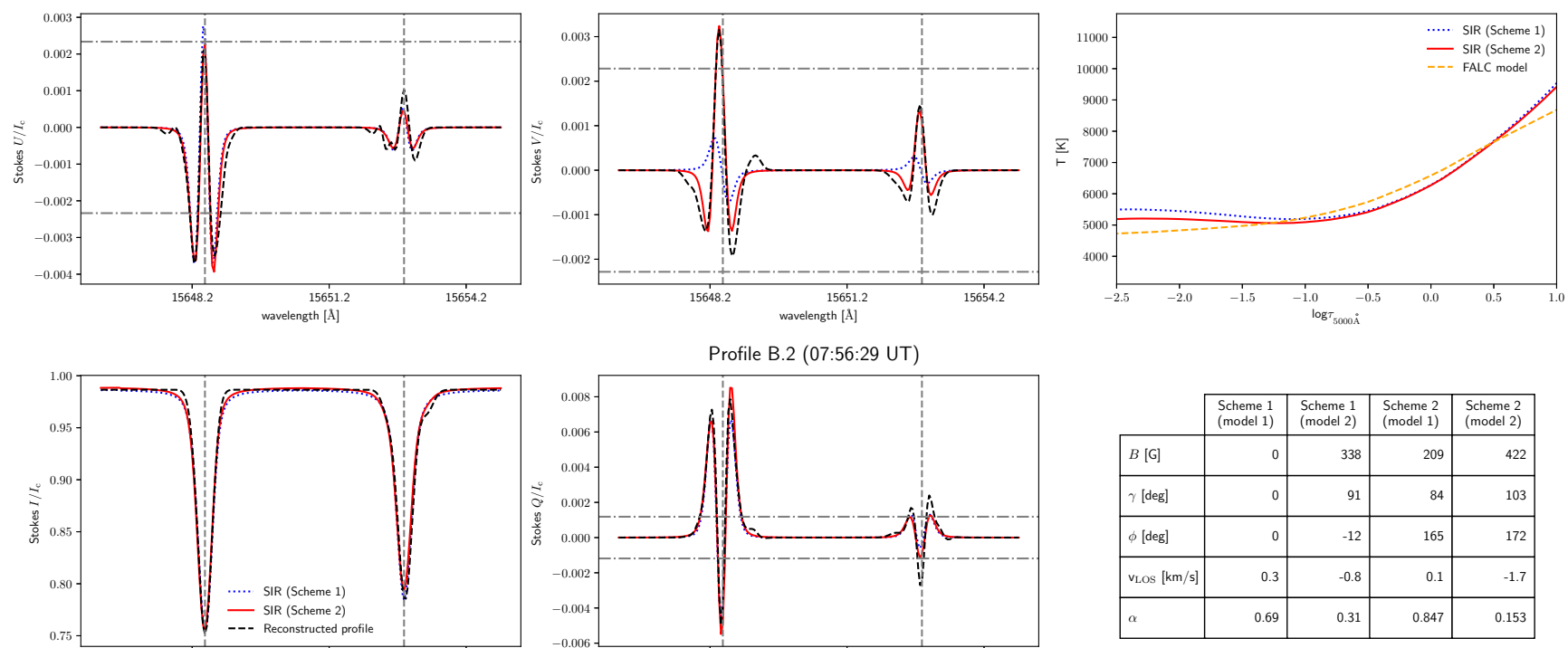

\begin{tabular}{|c|c|c|c|c|}
\hline & $\begin{array}{l}\text { Scheme } 1) \\
(\text { model 1) }\end{array}$ & $\begin{array}{l}\text { Scheme } 1 \\
(\text { model 2) }\end{array}$ & $\begin{array}{l}\text { Scheme } 1 \\
(\text { model 1 }\end{array}$ & $\begin{array}{c}\text { chheme } 2 \\
\text { (model 2) }\end{array}$ \\
\hline$B[G]$ & 0 & 338 & 209 & 422 \\
\hline$\gamma[\operatorname{deg}]$ & 0 & 91 & 84 & 103 \\
\hline$\phi[\operatorname{deg}]$ & 0 & -12 & 165 & 172 \\
\hline$v_{\operatorname{LOS}}[\mathrm{km} / \mathrm{s}]$ & 0.3 & -0.8 & 0.1 & -1.7 \\
\hline$\alpha$ & 0.69 & 0.31 & 0.847 & 0.15 \\
\hline
\end{tabular}
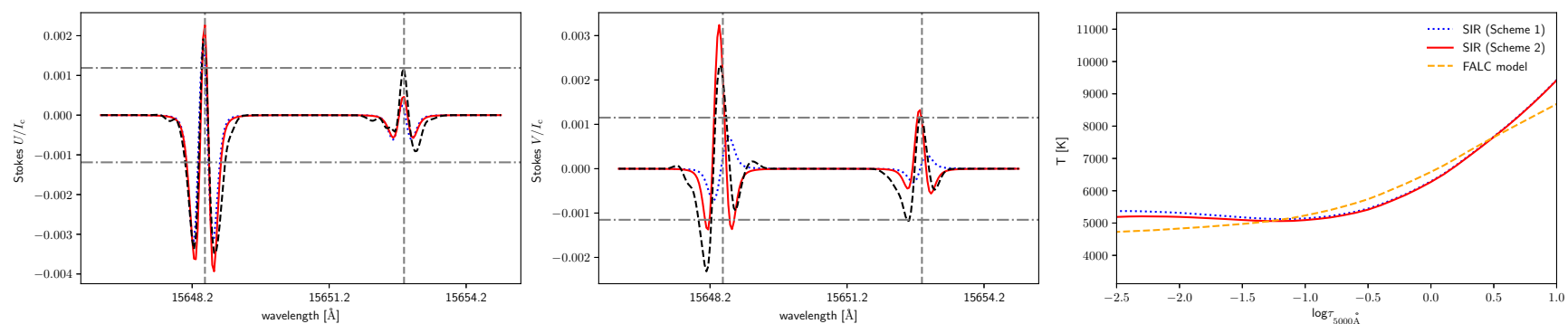

Fig. 2. Corrected Fig. 12.
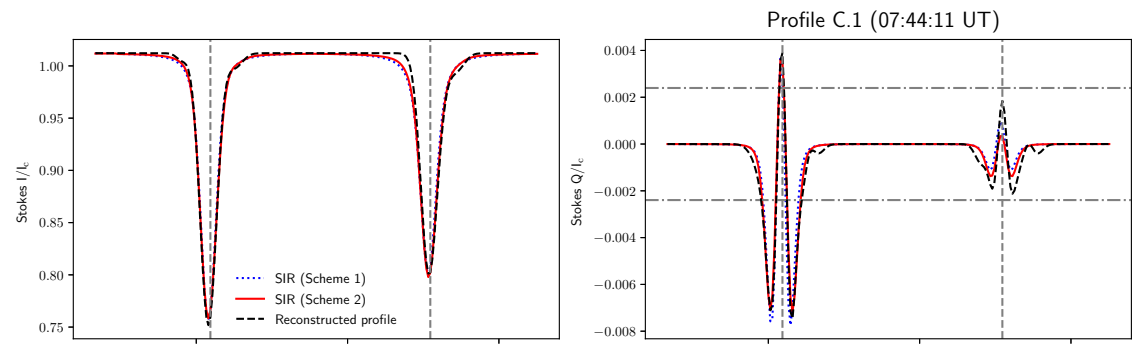

\begin{tabular}{|l|r|r|r|r|}
\cline { 2 - 5 } \multicolumn{1}{c|}{} & $\begin{array}{l}\text { Scheme 1 } \\
\text { (model 1) }\end{array}$ & $\begin{array}{l}\text { Scheme 1 } \\
\text { (model 2) }\end{array}$ & $\begin{array}{l}\text { Scheme 2 } \\
\text { (model 1) }\end{array}$ & $\begin{array}{r}\text { Scheme 2 } \\
\text { (model 2) }\end{array}$ \\
\hline$B[G]$ & 0 & 193 & 331 & 44 \\
\hline$\gamma$ [deg] & 0 & 85 & 89 & 55 \\
\hline$\phi[\operatorname{deg}]$ & 0 & 89 & 90 & 14 \\
\hline$v_{\text {L.OS }[\mathrm{km} / \mathrm{s}]}$ & -1.2 & -0.6 & -0.4 & -1.2 \\
\hline$\alpha$ & 0.239 & 0.761 & 0.378 & 0.622 \\
\hline$\alpha$
\end{tabular}
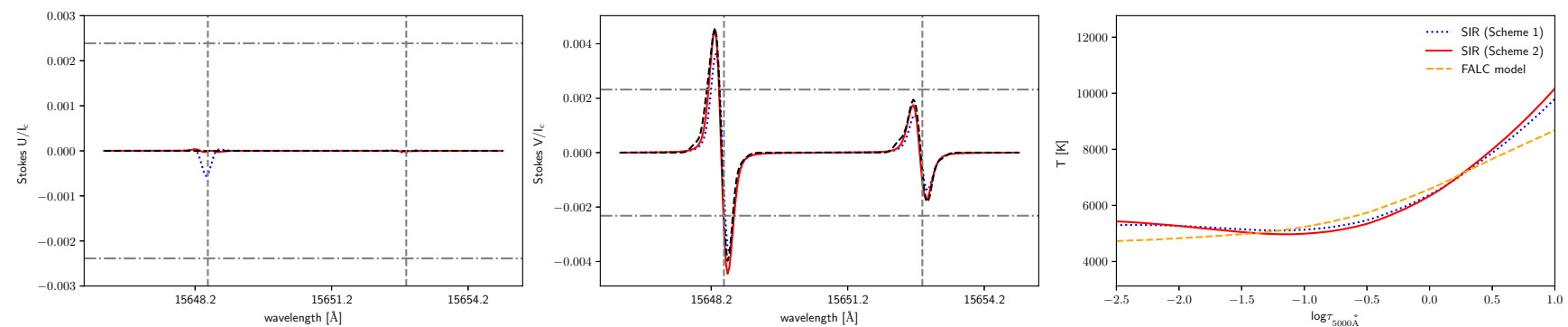

Fig. 3. Corrected Fig. 14. 
A\&A 652, C2 (2021)
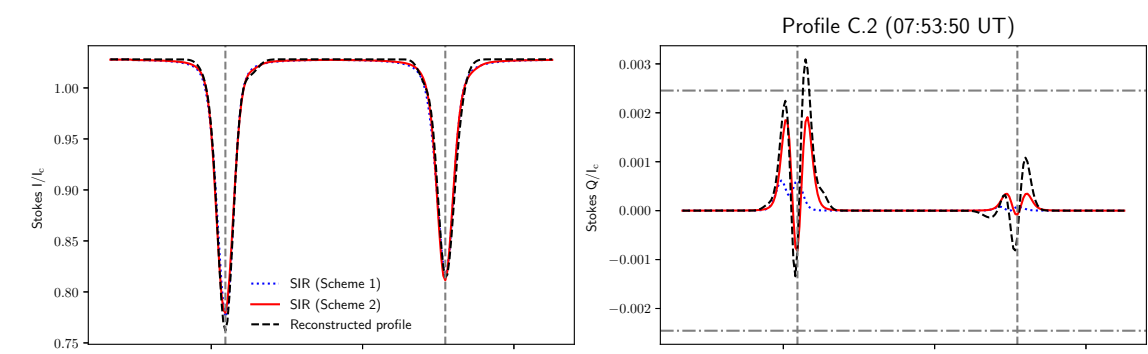

\begin{tabular}{|l|r|r|r|r|}
\cline { 2 - 5 } \multicolumn{1}{c|}{} & $\begin{array}{l}\text { Scheme 1 } \\
\text { (model 1) }\end{array}$ & $\begin{array}{l}\text { Scheme 1 } \\
\text { (model 2) }\end{array}$ & $\begin{array}{r}\text { Scheme 2 } \\
\text { (model 1) }\end{array}$ & $\begin{array}{r}\text { Scheme 2 } \\
\text { (model 2) }\end{array}$ \\
\hline$B[G]$ & 0 & 101 & 204 & 246 \\
\hline$\gamma[$ deg $]$ & 0 & 123 & 27 & 155 \\
\hline$\phi[$ [deg] & 0 & 6 & 183 & -3 \\
\hline$v_{\text {LoS }[\mathrm{km} / \mathrm{s}]}$ & 0.5 & -3.1 & -0.1 & -0.5 \\
\hline$\alpha$ & 0.805 & 0.195 & 0.542 & 0.458 \\
\hline
\end{tabular}
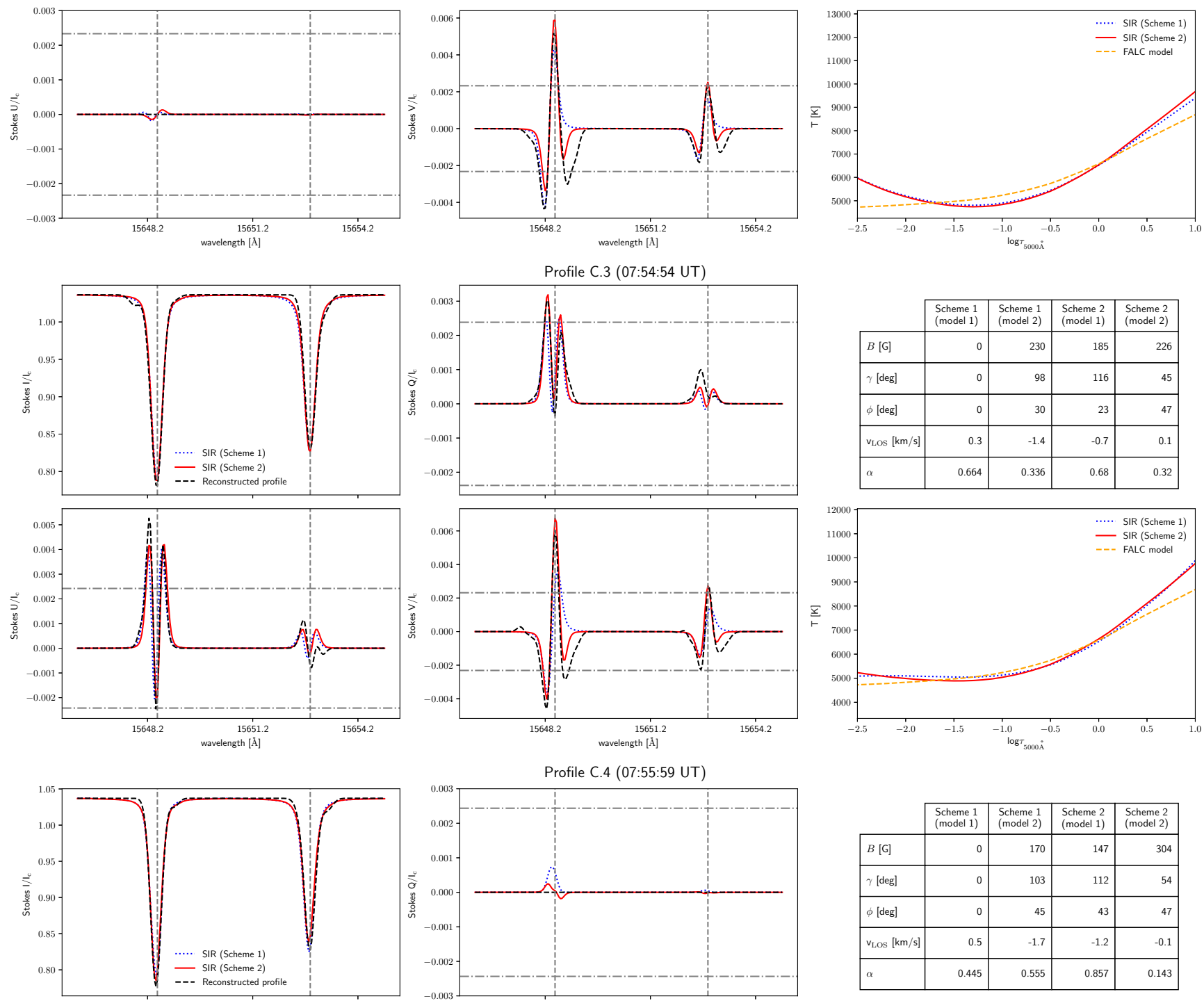

\begin{tabular}{|l|r|r|r|r|}
\cline { 2 - 5 } \multicolumn{1}{c|}{} & $\begin{array}{l}\text { Scheme 1 } \\
\text { (model 1) }\end{array}$ & $\begin{array}{l}\text { Scheme 1 } \\
\text { (model 2) }\end{array}$ & $\begin{array}{l}\text { Scheme 2 2 } \\
\text { (model 1) }\end{array}$ & $\begin{array}{l}\text { Scheme 2 } \\
\text { (model 2) }\end{array}$ \\
\hline$B[G]$ & 0 & 170 & 147 & 304 \\
\hline$\gamma$ [deg] & 0 & 103 & 112 & 54 \\
\hline$\phi[\operatorname{deg}]$ & 0 & 45 & 43 & 47 \\
\hline $\mathrm{v}_{\text {LoS }[\mathrm{km} / \mathrm{s}]}$ & 0.5 & -1.7 & -1.2 & -0.1 \\
\hline$\alpha$ & 0.445 & 0.555 & 0.857 & 0.143 \\
\hline$\alpha$
\end{tabular}
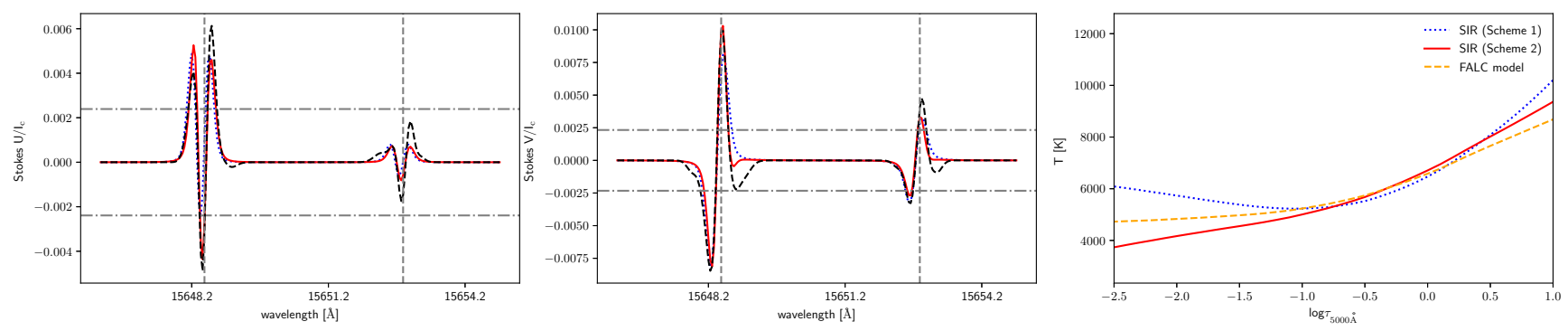

Fig. 4. Corrected Fig. 15. 
R. J. Campbell et al.: Temporal evolution of small-scale internetwork magnetic fields in the solar photosphere (Corrigendum)
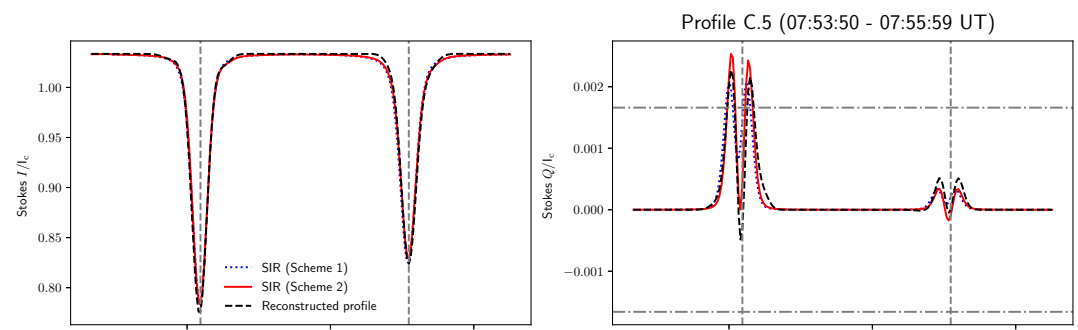

\begin{tabular}{|c|c|c|c|c|}
\hline & $\begin{array}{l}\text { Scheme 1 } \\
\text { (model 1) }\end{array}$ & $\begin{array}{l}\text { Scheme } 1 \\
\text { (model 2) }\end{array}$ & $\begin{array}{l}\text { Scheme 2 } \\
\text { (model 1) }\end{array}$ & $\begin{array}{l}\text { Scheme } 2 \\
(\text { model } 2)\end{array}$ \\
\hline$B[G]$ & 0 & 270 & 212 & 107 \\
\hline$\gamma$ [deg] & 0 & 105 & 114 & 45 \\
\hline$\phi[\mathrm{deg}]$ & 0 & 30 & 28 & 51 \\
\hline$v_{\text {LOS }}[\mathrm{km} / \mathrm{s}]$ & -0.0 & -1.7 & -0.9 & 0.3 \\
\hline & 0.802 & 0.198 & 0.532 & 0.468 \\
\hline
\end{tabular}
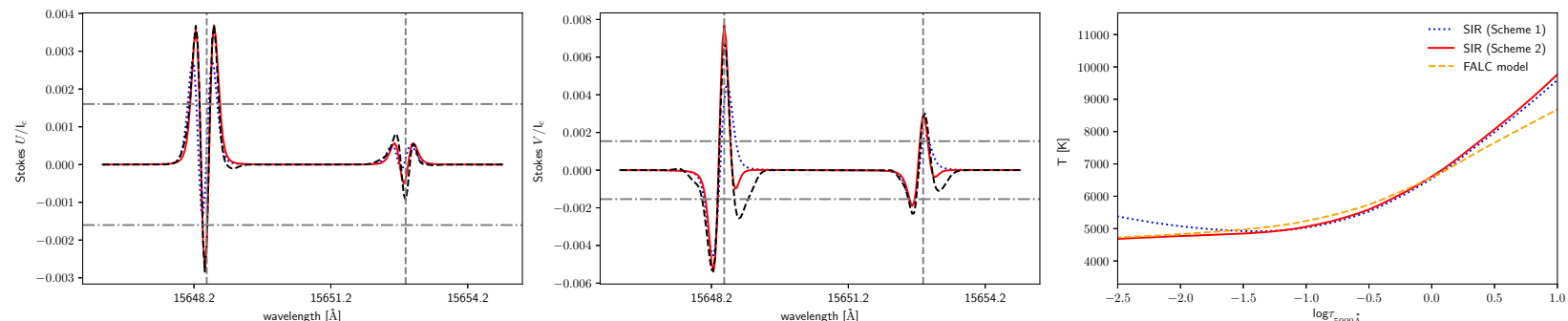

Fig. 5. Corrected Fig. 16. 